\title{
HIGHLIGHTS
}

\section{Targeting viruses in their phase}

4

better under-
standing of the
involvement
of LLPS during
the life cycle
of human
viruses could
result in the
development
of new antiviral
therapeutics

It is now well-established that weak, multivalent interactions involving proteins and nucleic acids can lead to the formation of membraneless macromolecular assemblies, or condensates, via liquid-liquid phase separation (LLPS). These condensates are prevalent in eukaryotic cells and we are now starting to appreciate their many cellular functions. LLPS has also been found to have roles in bacteria and during viral infection. In the latter case, LLPS may affect not only viral replication, but could also modulate host cell functions to promote viral spread. Hence, better understanding of the involvement of LLPS during the life cycle of human viruses could result in the development of new antiviral therapeutics. Two recent papers pave the way in this direction.

Wang et al. set out to study LLPS of the nucleocapsid protein of SARS-CoV-2 (SARS2-NP), which contains several intrinsically disordered regions (IDRs) - common drivers of LLPS. Indeed, SARS2-NP underwent LLPS both in vitro and in cells. This was, however, independent of IDRs, and instead mediated by the C-terminal dimerization domain.

Expression of SARS2-NP in cells blunted the activation of type I interferon (IFN) and the expression of IFN-responsive genes - key elements of the antiviral innate immune response, which is often hampered

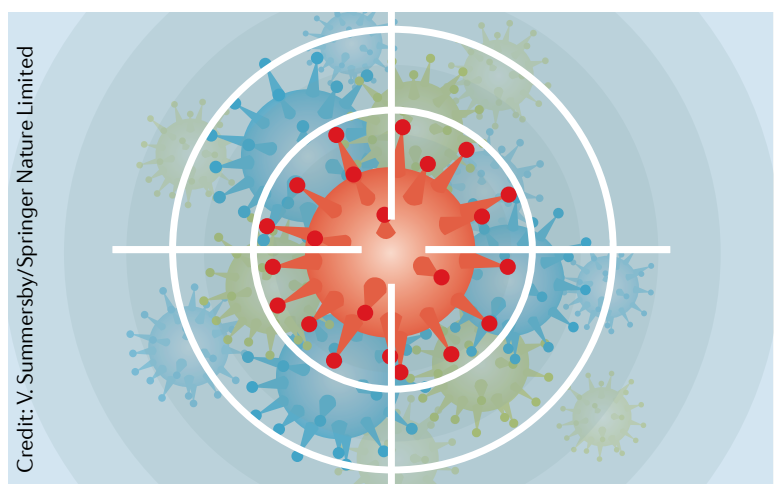

by SARS-CoV-2. Expression of SARS2-NP also potentiated the infectivity of another RNA virus, vesicular stomatitis virus (VSV), both in cells and in mice, in association with dampened type I IFN response. These inhibitory effects of SARS2NP on type I IFN signalling required SARS2-NP dimerization domain.

Type I IFN signalling is activated via viral RNA-induced formation of aggregates of mitochondrial antiviralsignalling protein (MAVS) - a process regulated by MAVS polyubiquitylation. SARS2-NP interacted with MAVS and hindered MAVS polyubiquitylation and aggregation in cells. This was negatively regulated by acetylation of Lys375 in SARS2-NP a residue adjacent to the dimerization domain - which inhibited SARS2-NP LLPS. This, together with the requirement for dimerization domain in blunting IFN signalling, indicates an important role for LLPS in modulating IFN antiviral response by SARS2-NP. However, how exactly SARS2-NP condensates modulate MAVS activity remains to be addressed.

Finally, the authors set out to design NP interfering peptides (NIPs) that modulate SARS2-NP LLPS by targeting the protein-protein interfaces in the dimerization domain. One of these peptides, NIP-V, was capable of inhibiting SARS2-NP LLPS and rescuing MAVS aggregation in cells. Importantly, pre-treatment of mice with NIP-V reduced the increased infectivity of VSV driven by co-expression of SARS2-NP and also reduced replication of SARS-CoV-2 itself, at the same time increasing serum levels of type I IFN in these mice. Hence, interfering with the LLPS of SARS2-NP could boost the immune response against SARS-CoV-2, reducing viral replication.

Risso-Ballester focused on a common-cold virus, respiratory syncytial virus (RSV), the replication of which relies on the formation of inclusion bodies. Inclusion bodies have been described as multiphasic condensates, containing an internal phase - inclusion body-associated granule (IBAG) - where M2-1 transcription factor and newly synthesized viral mRNAs are concentrated.

Inclusion bodies that form in RSV-infected cells indeed showed characteristics of dynamic, liquid-like condensates. Treatment with SonicHedgehog (SHH) antagonist cyclopamine (CPM, previously identified by the authors as an inhibitor of RSV replication), or with its $\mathrm{SHH}$-inactive derivative $\mathrm{A} 3 \mathrm{E}$, resulted in cessation of molecular dynamics in the inclusion bodies and their stabilization in cells. This suggested that the drugs affect the material properties of inclusion bodies, inducing the loss of liquid-like properties (promoting hardening). Their activity was linked to inclusion body structure and IBAG formation as CPM- or A3E-induced inclusion body hardening was abolished with a point mutation of M2-1 that yields RSV insensitive to CPM.

Importantly, mice treated with A3E showed dose-dependent inhibition of RSV replication, suggesting that inclusion body hardening has antiviral effect. As changes of inclusion body material properties induced by CPM/A3E occurred within minutes, treatment with condensate hardening drugs could become an efficient therapeutic strategy for acute viral infections where the speed of intervention is crucial.

Paulina Strzyz

ORIGINAL ARTICLES Wang, S. et al. Targeting liquid-liquid phase separation of SARS-CoV-2 nucleocapsid protein promotes innate antiviral immunity by elevating MAVS activity. Nat. Cell Biol. https://doi.org/10.1038/s41556-021-00710-0 (2021) | Risso-Ballester, J. et al. A condensatehardening drug blocks RSV replication in vivo. Nature https://doi.org/10.1038/s41586-02103703-z (2021)

RELATED ARTICLE Lyon, A. S., Peeples, W. B. \& Rosen, M. K. A framework for understanding the functions of biomolecular condensates across scales. Nat. Rev. Mol. Cell Biol. 22, 215-235 (2021) 